\title{
Effects of Vertical and Pinch Rolling on Residual Stress Distributions in Wire and Arc Additively Manufactured Components
}

\author{
Reza Tangestani, Gholam Hossein Farrahi, Mehdi Shishegar, Bahareh Pourbagher Aghchehkandi, Supriyo Ganguly, \\ and Ali Mehmanparast
}

(Submitted November 6, 2019; in revised form February 20, 2020; published online April 19, 2020)

\begin{abstract}
Residual stresses are inherent in parts manufactured using the wire + arc additive manufacturing (WAAM) technique, resulting in unpredictable mechanical response and structural integrity (Colegrove et al.: $J$ Mater Process Technol 213:1782-1791, 2013). An effective post-processing technique, which enhances the mechanical properties of WAAM parts, is rolling. This study investigates the vertical and pinch rolling effects on residual stress distribution in WAAM components. Initially, a WAAM model was created using a thermo-mechanical finite element modelling approach and validated against the experimental results. Subsequent to the validation of the model, the effect of the main parameters involved in vertical and pinch rolling processes, namely the rolling depth, the curvature depth of the roller, the roller shape, transversal displacement, rolling direction and roller thickness, was investigated. The results from this study show that the residual stress profile in the vertical rolling process applied on a WAAM wall can be enhanced by increasing the rolling depth and curvature depth of the roller. Moreover, it is shown that in the pinch rolling process, the residual stress profile is sensitive to the rolling direction and more compressive residual stresses can be induced into the wall by applying fewer passes of rolling using thicker rollers.
\end{abstract}

Keywords additive manufacturing, life enhancement, residual stress, rolling

\section{Introduction}

Additive manufacturing (AM) of large-scale functionally graded components requires an insight into the complicated microstructural features of these parts (Ref 1). Wire + arc additive manufacturing (WAAM) is by far the most efficient AM process with a relatively higher deposition rate $(1-4 \mathrm{~kg} / \mathrm{h})$, compared to other AM techniques, which enables the manufacturing of large parts up to several metres (Ref 2,3). This manufacturing process incorporates either gas metal arc welding (GMAW), plasma arc welding or gas tungsten arc welding techniques for metal deposition (Ref 3). Compared to its counterpart powder-based AM techniques, such as selective laser melting (SLM), the WAAM process offers a lower production cost due to its relatively high deposition rate (Ref 4). A wide range of weldable metals and alloys such as steel

Reza Tangestani and Gholam Hossein Farrahi, School of Mechanical Engineering, Sharif University of Technology, Tehran, Iran; Mehdi Shishegar, Mechanical Engineering Department, BuAli Sina University, Hamedan, Iran; and Bahareh Pourbagher Aghchehkandi, Fatigue Lab, School of Mechanical Engineering, Sharif University of Technology, Tehran, Iran; Supriyo Ganguly, Welding Engineering and Laser Processing Centre, Cranfield University, Cranfield, UK; and Ali Mehmanparast, Offshore Renewable Energy Engineering Centre, Cranfield University, Cranfield, UK. Contact e-mail: a.mehmanparast@cranfield.ac.uk.

\begin{tabular}{|ll|}
\hline & \multicolumn{1}{c|}{ Abbreviations } \\
\hline$a_{\mathrm{f}}$ & Length of the front ellipsoid \\
$a_{\mathrm{r}}$ & Length of the rear ellipsoid \\
$b$ & Length of the width ellipsoid \\
$c$ & Height of the ellipsoid \\
$f_{\mathrm{f}}$ & Factor of distributing the power for the front ellipsoid \\
$f_{\mathrm{r}}$ & Factor of distributing the power for the rear ellipsoid \\
$Q$ & Heat input \\
$q_{\mathrm{f}}$ & Input energy for the front ellipsoid \\
$q_{\mathrm{r}}$ & Input energy for the rear ellipsoid \\
$\mathrm{CD}$ & Curvature depth \\
$\mathrm{CHW}$ & Curvature half width \\
$\mathrm{HAZ}$ & Heat-affected zone \\
LD & Lateral direction \\
$\mathrm{RD}$ & Rolling depth \\
$\mathrm{TD}$ & Transversal displacement \\
WAAM & Wire + arc additive manufacturing \\
& \\
\hline
\end{tabular}

(Ref 5, 6), nickel (Ref 7) and titanium (Ref 8, 9) alloys can be employed in this technology. WAAM facilitates efficient manufacturing of Ti alloys such as Ti-6Al-4V alloy, which is extensively used in the aerospace industry. A substantial reduction in the buy-to-fly ratio from around 10-20 for a conventionally machined component to 1 could be achieved using this process (Ref 10). WAAM incorporates a multi-pass arc welding process, resulting in the efficient manufacturing of large-scale parts with acceptable mechanical properties at a lower production cost (Ref 2).

Despite the numerous advantages of WAAM, the mechanical properties of the final parts display unpredictable mechanical properties due to the process-induced residual stresses and 
changes in the microstructure (Ref 11). Moreover, a complex thermal stress distribution from the repeated depositioning process gives rise to high residual distortion and subsequently crack formation in some cases (Ref 12). Recent studies have explored various post-processing techniques to enhance the mechanical properties of WAAM parts, such as shot peening and high-depth rolling, as well as controlling the build path (Ref 4). Limited research studies have focused on experimental and numerical investigations of residual stresses in WAAM parts. In these limited studies, it has been shown that measuring residual stresses in WAAM components using experimental techniques imposes constraints and cost implications (Ref 13). Numerical simulations, however, facilitate the prediction of residual stresses across all regions in the WAAM parts. In most of the simulations, a heat source moves and elements are built along the travelling direction (Ref 14-17).

Due to the nonlinear material models used in these simulations, the numerical analysis on WAAM components is often relatively time-consuming. Very limited research is available in the literature to examine residual stress optimization, reducing tensile damaging and locked-in stresses in WAAM parts. Although previous studies have proposed appropriate techniques to reduce residual stresses, it is still unclear how these stress reduction techniques could be improved and optimized further. Colegrove et al. (Ref 1) showed that using a slotted roller shape was more beneficial compared to the conventional roller used in WAAM parts as it can protect the created wall from lateral distortion and transfer the applied load in a more efficient and uniform manner. However, the effect of other roller shapes (e.g., pinch and vertical rolling) on the residual stress has not yet been investigated. Indeed, performing a parametric study on the influence of rolling on residual stress profiles in additive manufactured structures by means of finite element modelling is an efficient and reliable approach to develop a comprehensive understanding of local stress distributions across the entire volume of the structure.

The main aim of this study is to numerically examine different roller shapes' and geometric parameters' effects on residual stress profiles in the WAAM parts. A three-dimensional (3D) WAAM model has been created in this study to predict residual stresses in AM parts which have been validated through comparison with the experimental results presented in Ref 5. Moreover, various techniques to reduce the tensile damaging and residual stresses have been reviewed, and recommendations are provided for residual stress enhancement in WAAM parts using each of these techniques.

\section{Material Specification of the WAAM Wall}

The sample bedplate (AKA baseplate) in this study was made of S355JR rolled structural steel (see Fig. 1), the chemical composition of which is summarized in Table 1. This material is widely used in industry, particularly for oil \& gas and offshore wind turbine applications. The chemical composition of the consumable electrode (Fe balance) is summarized in Table 2. This material mainly consists of austenite and ferrite phases, where the former has a negligible influence on the material properties. Therefore, material phase transformation modelling is not required to be considered in numerical simulations.

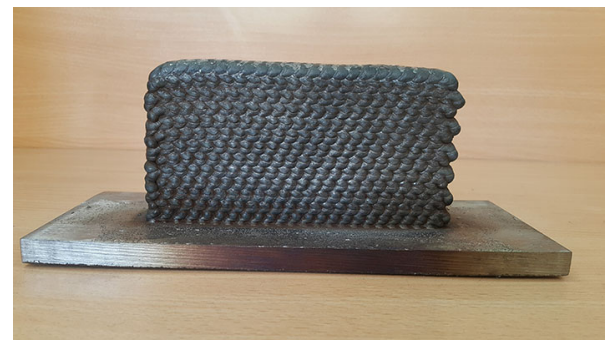

Fig. 1 An example of a WAAM sample fabricated using S355JR steel baseplate

Table 1 Chemical composition of the S355JR baseplate (in wt.\%)

\begin{tabular}{lcccccc}
\hline $\mathbf{C}$ & $\mathbf{M n}$ & $\mathbf{S i}$ & $\mathbf{P}$ & $\mathbf{S}$ & $\mathbf{N}$ & $\mathbf{N b}$ \\
\hline 0.24 & 1.60 & 0.55 & 0.045 & 0.045 & 0.009 & $0.003-0.100$
\end{tabular}

Table 2 Chemical composition of the consumable electrode (in wt.\%)

\begin{tabular}{lccccc}
\hline C & Mn & Si & Cu & P & S \\
\hline 0.08 & 1.50 & 0.92 & 0.16 & $\leq 0.040$ & $\leq 0.035$ \\
\hline
\end{tabular}

The welding wire diameter used in the fabrication of the WAAM wall considered in this study was $1.2 \mathrm{~mm}$, with the wire feed speed of $10 \mathrm{~m} / \mathrm{min}$ and the welding torch travel speed of $8.33 \mathrm{~mm} / \mathrm{s}$. The welding process heat input was $269.5 \mathrm{~J} / \mathrm{mm}$, with the waiting time of $400 \mathrm{~s}$ between subsequent layers in order to cool down the deposited layer to below $50{ }^{\circ} \mathrm{C}$ before new layers were deposited (Ref 5). A water-cooled aluminium backing plate was employed to facilitate the cooling process.

\section{Finite Element Model Set-up for WAAM Simu- lation and the Rolling Process}

\subsection{Thermo-Mechanical Finite Element Modeling}

A deposited wall with geometry similar to that of Ref 5 was built in ABAQUS to study the rolling process (see Fig. 1). To reduce the computational time, a selected part with a shorter length, in the $X$ direction, shown in Fig. 2, was modelled. The cooling plate, modelled as a rigid plane of $200 \times 100 \mathrm{~mm}^{2}$ with a mass of $1 \mathrm{~kg}$, was also defined in the finite element model set-up to prevent the bedplate nodes from moving in the $Z$ direction during the thermo-mechanical finite element analysis.

The "element birth technique" (Ref 15, 19) was used to simulate deposition of the layers in the finite element simulation. In order to do so, all the wall elements were deactivated in the first step and then activated sequentially by following the arc movement. Each layer was built in $12 \mathrm{~s}$ with a $400 \mathrm{~s}$ pause between depositioning of the consecutive layers. The pause enabled the sample to cool down to below $100{ }^{\circ} \mathrm{C}$ before new 


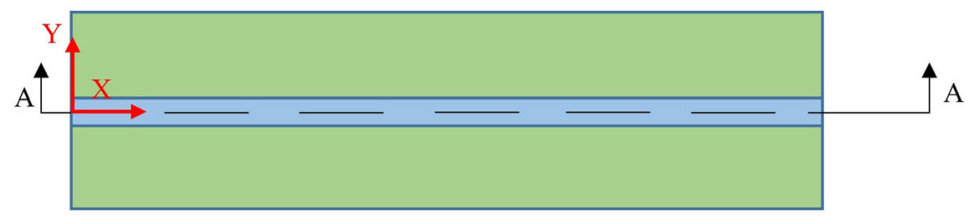

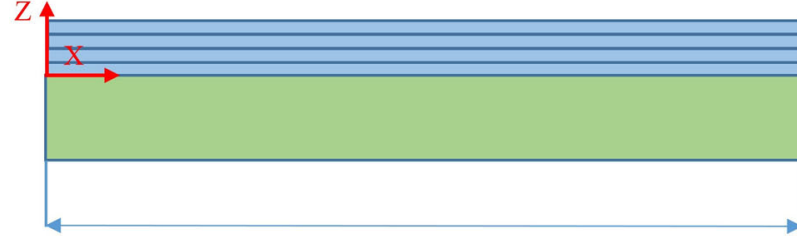

100

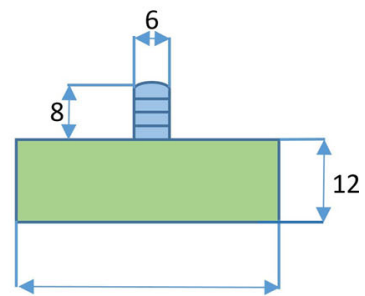

60

Fig. 2 The modelled geometry in finite element analysis. All dimensions are in millimetres

layers were deposited. To model the heat flux for the thermal simulation, the Goldak approach (Ref 20) was employed in the simulations using the following equations:

$q_{\mathrm{f}}=\frac{6 \sqrt{3} Q f_{\mathrm{f}}}{\pi \sqrt{\pi} a_{\mathrm{f}} b c} e^{\left[-3\left(\frac{x^{2}}{a_{\mathrm{f}}^{2}}+\frac{y^{2}}{b^{2}}+\frac{z^{2}}{c^{2}}\right)\right]}$

$q_{\mathrm{r}}=\frac{6 \sqrt{3} Q f_{r}}{\pi \sqrt{\pi} a_{\mathrm{r}} b c} e^{\left[-3\left(\frac{x^{2}}{a_{\mathrm{r}}^{2}}+\frac{y^{2}}{b^{2}}+\frac{z^{2}}{c^{2}}\right)\right]}$

$f_{\mathrm{f}}+f_{\mathrm{r}}=2$

$\frac{f_{\mathrm{f}}}{f_{\mathrm{r}}}=\frac{a_{\mathrm{f}}}{a_{\mathrm{r}}}$

where $f_{\mathrm{r}}$ and $f_{\mathrm{r}}$ are the fractions of the heat deposited in the front and rear quadrants, $q_{\mathrm{f}}$ is the power density distribution inside the front ellipsoid $\left(\mathrm{W} / \mathrm{m}^{3}\right), q_{\mathrm{r}}$ is the power density distribution for the rear ellipsoid $\left(\mathrm{W} / \mathrm{m}^{3}\right), a_{\mathrm{f}}$ is the length of the front ellipsoid, $a_{\mathrm{r}}$ is the length of the rear ellipsoid, $b$ is the length of the width ellipsoid, and $c$ is the height of the ellipsoid. Equations 1 and 2 are employed to define the power density distribution for the front and rear ellipsoids, where $x, y$ and $z$ are the longitudinal, lateral and normal dimensions, respectively. The Goldak model incorporates two different ellipsoidal equations with distinct parameters, the values of which are summarized in Table 3.

The mechanical and thermal properties of the material examined in this work were taken from the data available in Forni et al. (Ref 21). Following the proposed numerical framework in Ref 5, the convection coefficient and emissivity in finite element simulation models were assumed to be independent of the temperature and were set to $5.7 \mathrm{~W} \mathrm{~m}^{-2} \mathrm{~K}^{-1}$ and 0.2 , respectively. A convection coefficient of $300 \mathrm{~W} \mathrm{~m}^{-2} \mathrm{~K}^{-1}$ (Ref 5) was taken for the cooling system, which is located under the bedplate. The heat-affected zone (HAZ) convection appeared to be higher compared to the other layers, resulting in a curve-shaped geometry in the last layer.

The element size was controlled by the rolling model, as a significant plastic region was formed under the roller movement, which required mesh refinement for an accurate analysis. The element size in the wall was required to be less than $1 \mathrm{~mm}$ in $X, 0.6 \mathrm{~mm}$ in $Y$ and $0.8 \mathrm{~mm}$ in the $Z$ direction, based on the
Table 3 Parameters used in the Goldak model (Ref 5, 20)

\begin{tabular}{lcccccc}
\hline $\boldsymbol{Q}, \mathbf{W}$ & $\boldsymbol{f}_{\mathbf{f}}$ & $\boldsymbol{f}_{\mathbf{r}}$ & $\boldsymbol{b}, \mathbf{m m}$ & $\boldsymbol{c}, \mathbf{m m}$ & $\boldsymbol{a}_{\mathbf{f}}, \mathbf{m m}$ & $\boldsymbol{a}_{\mathbf{r}}, \mathbf{m m}$ \\
\hline 2245.83 & 0.6 & 1.4 & 2.5 & 3 & 2 & 6 \\
\hline
\end{tabular}

mesh sensitivity results. The best aspect ratio for the element size was found to be one, which resolved the convergence issue and gave accurate results. The selected mesh for the wall consisted of cubic elements, with 180, 5 and 16 elements in the $X, Y$ and $Z$ directions, respectively (see Fig. 3). The mesh size was doubled in the $X$ and $Z$ directions in the bedplate to reduce the computational time for completing these simulations (see Fig. 3). This change in the element size did not affect the final results, as the elements in this region were fixed by clamps.

\subsection{Validation of the Thermo-Mechanical Model}

A comparison between the predicted data obtained from the model developed in this study and the experimental residual stress measurement results of Ding et al. (Ref 5) is presented in Fig. 4. It can be seen in this figure that an abrupt change in the residual stress profile is observed in the region where the wall was deposited on the bedplate. It is worth noting that as a result of thermal input, the stress generated throughout the entire wall was approximately $500 \mathrm{MPa}$. The overall observation in Fig. 4 is that a tensile residual stress profile of between around 400 and $550 \mathrm{MPa}$ along the longitudinal direction remains in the WAAM wall subsequent to deposition of the final layer. As seen in this figure, the predicted results are in very good agreement with the experimental data, indicating that the developed numerical model provides accurate predictions of residual stresses. The created model also reduced the computational time by about $90 \%$.

\subsection{Numerical Framework for Simulating the Rolling Process}

In order to investigate the effect of roller shapes and geometric parameters on the induced residual stresses in WAAM parts, a finite element model has been developed in ABAQUS to simulate the vertical rolling and pinch rolling processes that are schematically shown in Fig. 5. The details of the model set-up are explained below. 


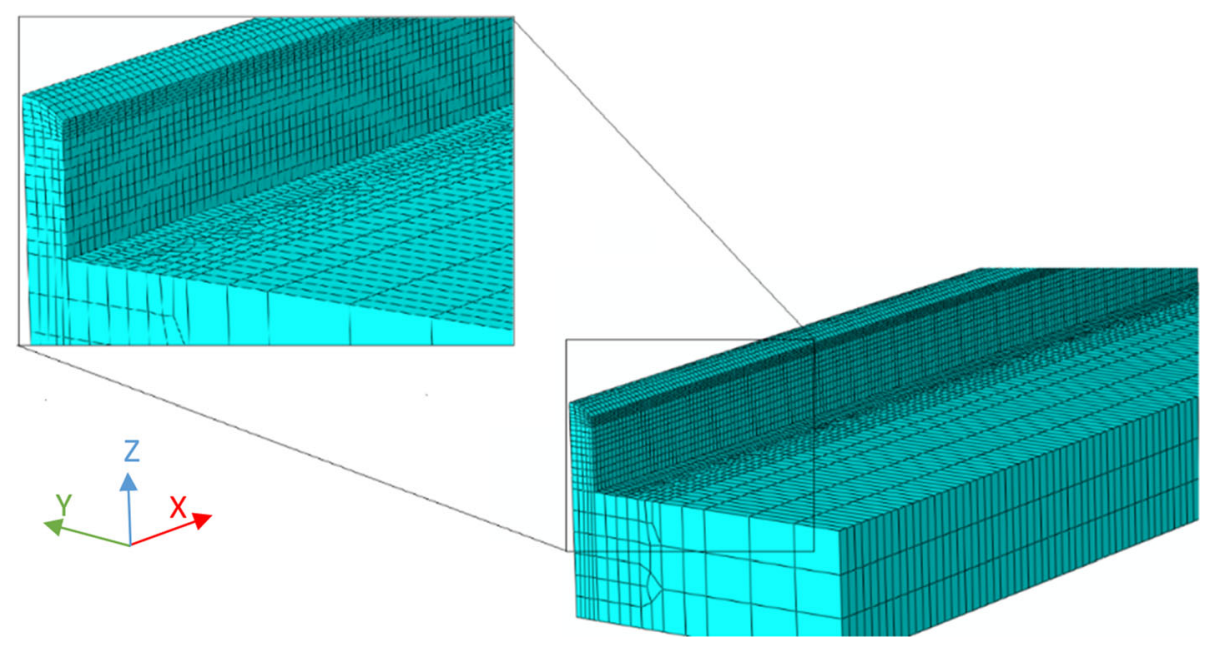

Fig. 3 The mesh design in the WAAM simulations

3.3.1 Rolling Process Parameters. A roller radius of $5 \mathrm{~cm}$ with a travel speed of $50 \mathrm{~cm} / \mathrm{min}$, which is typically used in the WAAM rolling process, has been considered and modelled in finite element simulations. In the numerical analysis, the roller has been defined as a rigid body due to negligible deformation in the roller compared to the WAAM wall. The rolling process was applied after the fourth cooling step. To induce plastic strain due to the rolling process on the top of the WAAM wall, a curvature at the contact surface was incorporated to engage with the convex crown (Ref 5). It is known that the magnitude of the applied load and the geometry of the roller curvature significantly affect the rolling process.

3.3.2 Vertical Rolling Process. The WAAM parts are rolled using two types of vertical roller-profiled and slotted-where the former is engaged with the convex crown of the bedplate, while the latter incorporates an additional deep slot to restrain the lateral distortion of the wall (see Fig. 6). One profiled roller (Fig. 6a; type A) and two types of slotted profile roller (Fig. 6b; type B and Fig. 6c; type C) were modelled in this study. One slotted roller was created using a curved contact surface (see Fig. 6b; type B), while the other had a flat surface contact (see Fig. 6c; type C). According to the procedures described in the literature, the vertical rolling can be applied after or before the cooling step and after the deposition of each layer.

In the vertical rolling process, the rolling depth (RD) is known to be the most significant parameter, which is controlled by the amount of force applied on the roller (see Fig. 7). To investigate the residual stress distribution in numerical simulations, the $\mathrm{RD}$ values of $0.1,0.2,0.5,0.75$ and $1 \mathrm{~mm}$ have been considered in the analyses.

In the case of the profiled roller, which is schematically shown in Figs. 6(a) and 8, the curvature half width (CHW) of the roller was taken as $2.6 \mathrm{~mm}$ (see Fig. 8). Moreover, a transition fillet radius of $0.1 \mathrm{~mm}$ between the curvature and the outer surface was considered in numerical simulations using the profiled roller. It is worth noting that the CHW parameter requires to be greater than half of the WAAM wall thickness in order to enable the roller to engage with the top layer of the WAAM wall. Five different profiled roller geometries were modelled to study the effect of the curvature depth (CD) parameter, ranging from 1 to $2.5 \mathrm{~mm}$, on the induced residual stress fields. For the $\mathrm{CD}$ value of $1 \mathrm{~mm}$, the roller concave

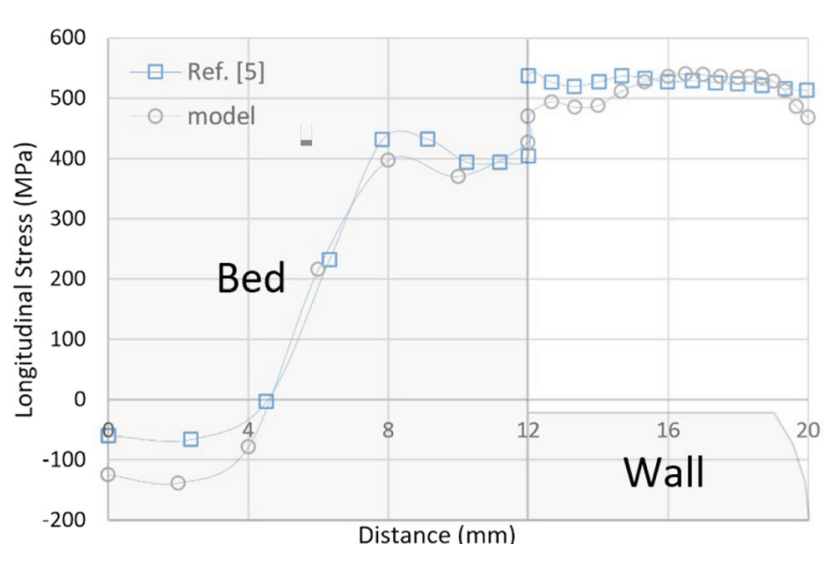

Fig. 4 Longitudinal residual stresses in the designated path before the unclamping step, obtained from the present study compared with the experimental results in Ref 5

crown was similar to the convex crown of the last deposited layer in the WAAM wall.

3.3.3 Pinch Rolling Process. According to the previous studies found in the literature, pinch rolling (see Fig. 5b) enables the smoothing of the increased surface waviness caused by vertical rolling (Ref 18). Moreover, this rolling technique is known to be more efficient and quicker compared to the vertical rolling technique, due to the fact that the load can be simply increased without increasing the wall waviness. In this study, a travel speed of $50 \mathrm{~cm} / \mathrm{min}$ and a flat contact surface were assumed and the transversal displacement (TD), lateral direction (LD) and roller thickness parameters were investigated.

The TD parameter was investigated by building a model for three roller passes (see Fig. 9) and examination of three types of rollers with a TD of 3, 3.5 and $4 \mathrm{~mm}$. The thickness, roller radius and RD in these simulations were 4,50 and $0.1 \mathrm{~mm}$, respectively. It was noted that multiple rolling operations were required when the roller was thinner than the wall height, resulting in a gap between passes.

The LD is a rolling process parameter that becomes particularly crucial when the number of passes is greater than one. This is known to be due to the bedplate constricting the wall from the bottom, which influences the residual stress distribution. Pinch rolling was performed either from top to 


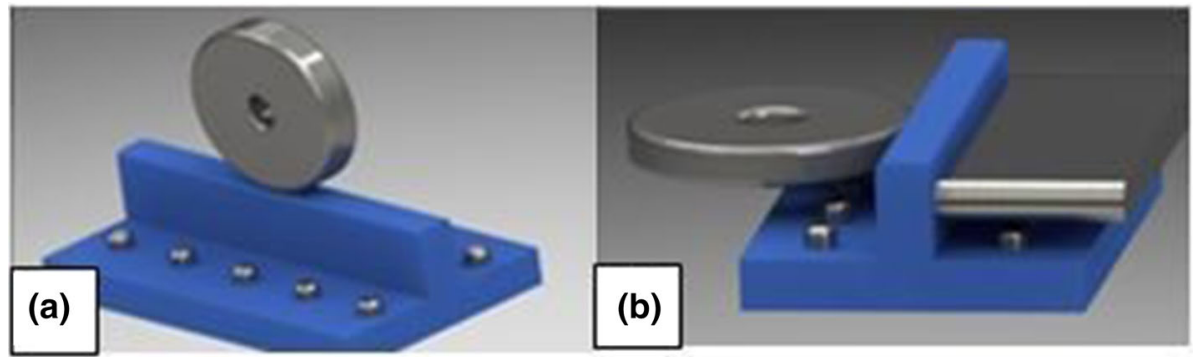

Fig. 5 Schematic demonstration of (a) vertical rolling and (b) pinch rolling

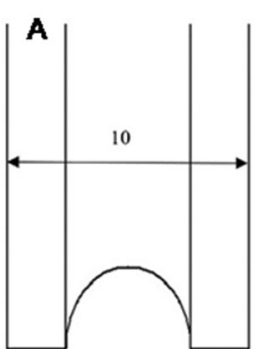

(a)

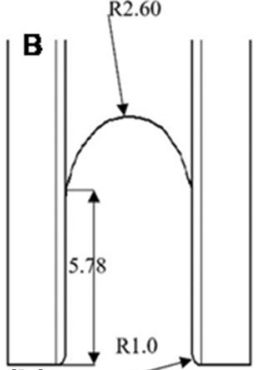

(b)

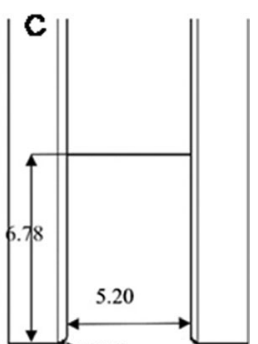

(c) $\mathrm{R} 1.0$

Fig. 6 Demonstration of different roller shapes: (a) profiled roller; type A, (b) curved-slotted roller; type B, and (c) flat-slotted roller; type C. All dimensions are in $(\mathrm{mm})$

bottom or in the opposite direction (see Fig. 9). This was performed with a TD of $2 \mathrm{~mm}$ in both cases. A rolling thickness of $4 \mathrm{~mm}$ with a $0.4-\mathrm{mm}$ fillet at the edges and an RD of $0.1 \mathrm{~mm}$ were used for this simulation.

The roller thickness was also studied by considering three different thicknesses of 4, 6 and $8 \mathrm{~mm}$. Although larger rollers cover a wider contact area, therefore speed up the rolling process, these rollers could not be larger than the wall height, as this would impede the uniform load transfer on the wall. When the larger roller was used, a single rolling step was sufficient; however, two and three rolling operations were needed to cover a wall height of $8 \mathrm{~mm}$ when medium and smaller rollers were employed in the simulations. The rolling process was applied from the bottom to top with a TD of $2 \mathrm{~mm}$ which was defined between the rolling passes for the medium and smaller rollers.

3.3.4 Rolling Simulation on the WAAM Wall. The first step in simulating the rolling process was to transfer the generated residual stress data from the WAAM simulation to the rolling model. In the second step, the quasi-static movement of the roller was modelled. In the third step, all the boundaries were removed apart from the symmetry boundary condition and a fixed node was implemented to prevent the rigid body from having any movement. Finally, the predicted residual stress profiles were obtained from the mid-length of the simulated WAAM part (see Fig. 10).

\section{Residual Stress Prediction Results and Discus- sion}

Finite element simulations have been performed to predict vertical and pinch rolling effects on residual stress profiles at the mid-length of the WAAM built wall (see Fig. 10b), and the

results are presented in this section. The overall observation is that in all simulations, the amount of tensile residual stress at the rolled surface is close to the yield stress of the material, which is consistent with the observations reported in Ref 22 .

\subsection{Vertical Rolling Process}

4.1.1 Rolling Depth Effects on Residual Stresses. The influence of different RDs on residual stress distributions in the WAAM wall has been investigated, and the results are shown in Fig. 11 and 12 for longitudinal stresses (i.e., along the $X$ axis) and normal stresses (i.e., along the $Z$ axis), respectively. Also included in these figures are the results from the simulation without any rolling (i.e., non-rolled). In Fig. 11 and 12 and the following figures, the residual stress variation is plotted against the WAAM wall height (i.e., measured from the bottom to the top of the wall as schematically shown in Fig. 3). As seen in Fig. 11, the largest magnitude of compressive residual stress in the longitudinal direction is found at $6 \mathrm{~mm}$ wall height when $0.2 \mathrm{~mm}$ RD was applied. The results in Fig. 11 show that at this wall height, the residual stress values for $0.1,0.2$ and $0.5 \mathrm{~mm}$ RDs have been found to be compressive, while tensile values of residual stresses are predicted for greater RDs. It can be seen in Fig. 12 that the largest magnitude of compressive residual stress in the normal direction is found to be at the mid-height of the wall (i.e., at approximately $4 \mathrm{~mm}$ wall height) which corresponds to the RD of $1 \mathrm{~mm}$. This figure clearly shows that the magnitude of beneficial compressive residual stress increases by increasing the RD. The results show that by applying a larger value of $\mathrm{RD}$, more pressure is induced on the top layers, which leads to the creation of a larger plastic region and subsequently a greater penetration depth for compressive normal stresses in the WAAM wall.

Comparison of the RD effects on the predicted residual stress profiles shows that by increasing the value of RD from 0.1 to $1 \mathrm{~mm}$, the magnitude of compressive normal stress increases by $43 \%$. Furthermore, the predicted residual stress profiles in Fig. 12 show that increasing the RD value increases the penetration depth of the compressive residual stresses along the normal direction to the lower layers of the WAAM wall. An increase in the wall deformation was also observed due to the larger force applied to the top of the wall. Also, it is seen in Fig. 12 that the peak tensile residual stress along the normal direction was decreased by increasing the RD value with relatively lower stress gradients from tensile to compressive stress fields. This indicates that increasing the RD enhances the fatigue life of the WAAM components by inducing a deep compressive residual stress field in the manufactured part. 


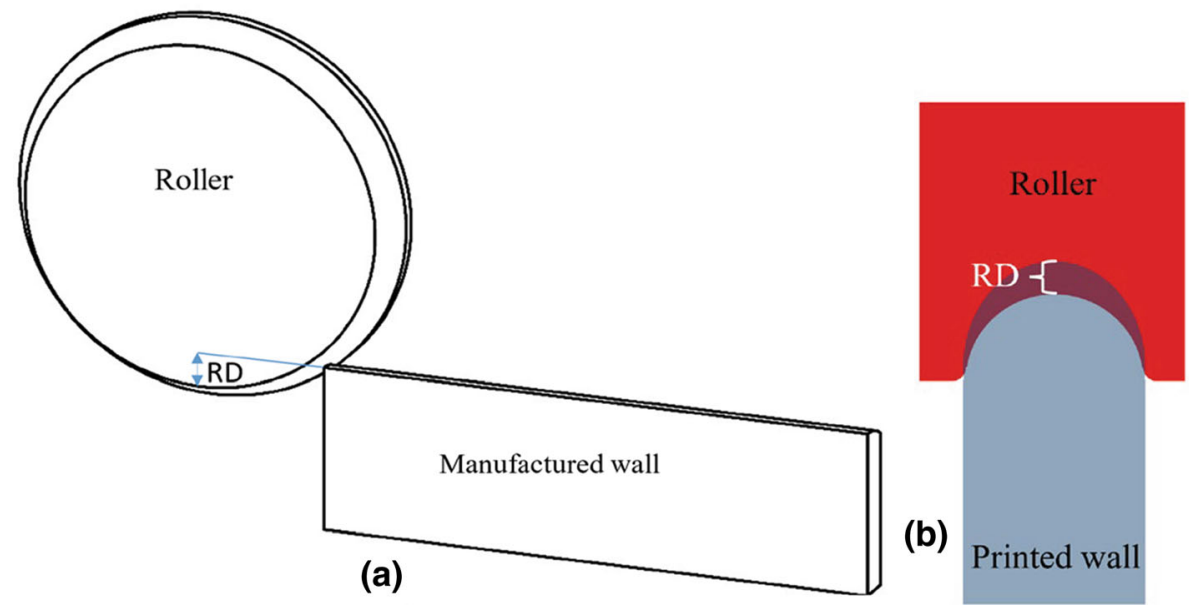

Fig. 7 Roller position at the beginning of the process: (a) side view, (b) cross-sectional view

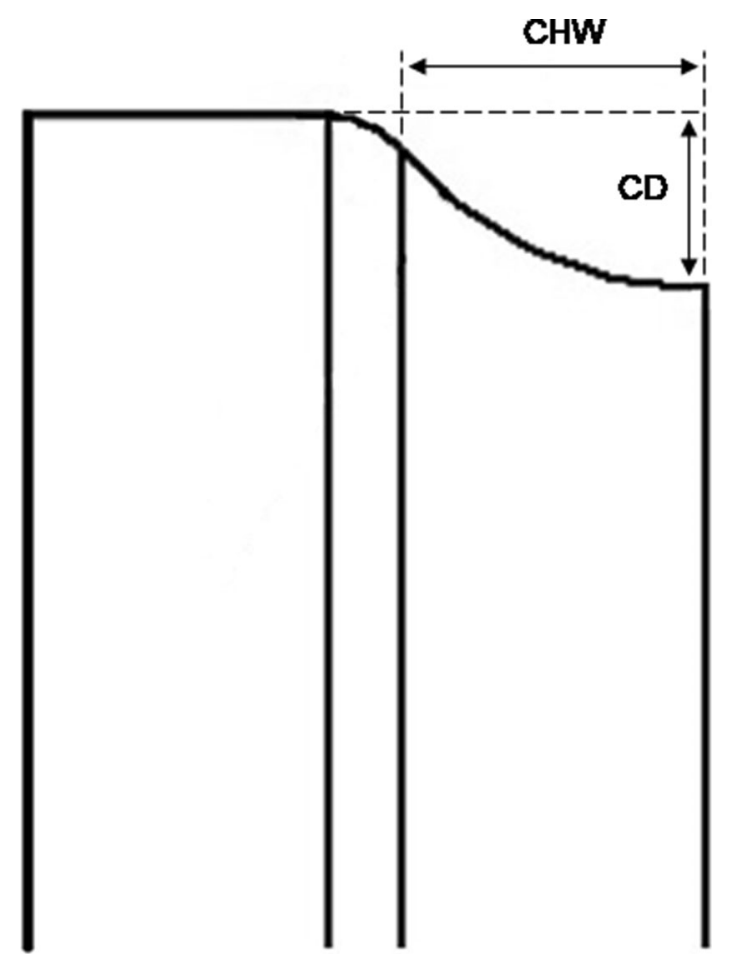

Fig. 8 Key dimensions in the profiled roller geometry

4.1.2 Curvature Depth. The influence of different CDs of $1,1.5,2$ and $2.5 \mathrm{~mm}$ on the induced residual stresses in the WAAM wall has been investigated, and the results for longitudinal stresses (i.e., along the $X$ axis) and normal stresses (i.e., along the $Z$ axis) are shown in Fig. 13 and 14, respectively. Also included in these figures are the results from the simulation without any rolling. As seen in both figures, the $\mathrm{CD}$ effect on longitudinal and normal stresses is found to be insignificant near the contact surface. However, the penetration depth of compressive stresses increases by increasing the $\mathrm{CD}$, particularly in normal stress. It is worth noting that a curvature with a larger depth can effectively restrict the deposition of a multilayered structure.
The normal stress results in Fig. 14 show that, depending on the $\mathrm{CD}$, tensile residual stresses of similar magnitudes can be formed in the wall height of up to around $4 \mathrm{~mm}$. Moreover, the results show that increasing the $\mathrm{CD}$ minimizes the extent of tensile-damaging residual stresses in the bottom layers of the WAAM wall. It can be seen in Fig. 14 that rolling with a larger $\mathrm{CD}$ reduces the stress more effectively, exhibiting a semicircular curve which enables transmitting the rolling effects deeper through the WAAM part. As an example, for only a $1.5 \mathrm{~mm}$ change in the CD from 1 to $2.5 \mathrm{~mm}$, the effective penetration depth of the compressive residual stress is increased by about $50 \%$. For the same amount of increase in the CD value, the maximum tensile stress was only changed by approximately $10 \mathrm{MPa}$ in the bottom layers. For the regions near to the contact surface, however, the results were almost unchanged.

In general, the results show that increasing the $\mathrm{CD}$ value leads to an increase in the compressive residual stress penetration depth in a normal direction (i.e., along the $Z$ axis) which is desirable as it compensates for the tensile residual stress created during the WAAM process. This indicates that the fatigue life of the WAAM, particularly at the top layers, can be enhanced by increasing the $\mathrm{CD}$ during the rolling process.

4.1.3 Profiled and Slotted Rollers. The residual stress prediction results for different types of roller, shown in Fig. 6, along longitudinal and normal directions, are presented in Fig. 15 and 16, respectively. Figure 15 shows that a comparatively larger area of compressive residual stress penetration depth as well as the minimum longitudinal stress of approximately $-350 \mathrm{MPa}$ is achieved using roller $\mathrm{B}$, making it the most effective choice compared to roller types $\mathrm{A}$ and $\mathrm{C}$. Comparing the other two roller types, roller A appears to be more effective as it exhibits a larger magnitude and penetration depth for compressive longitudinal stress compared to roller C. It must be noted that the shape of the roller affects the lateral deformation in the WAAM part (Ref 23). In the current study, the lateral deformation was found to be almost entirely eliminated using rollers $\mathrm{B}$ and $\mathrm{C}$, unlike roller $\mathrm{A}$ which resulted in significant lateral deformation.

The normal stress results in Fig. 16 show that roller B induces compressive normal stresses in a deeper proportion of the deposited layers with the highest magnitude of compressive 


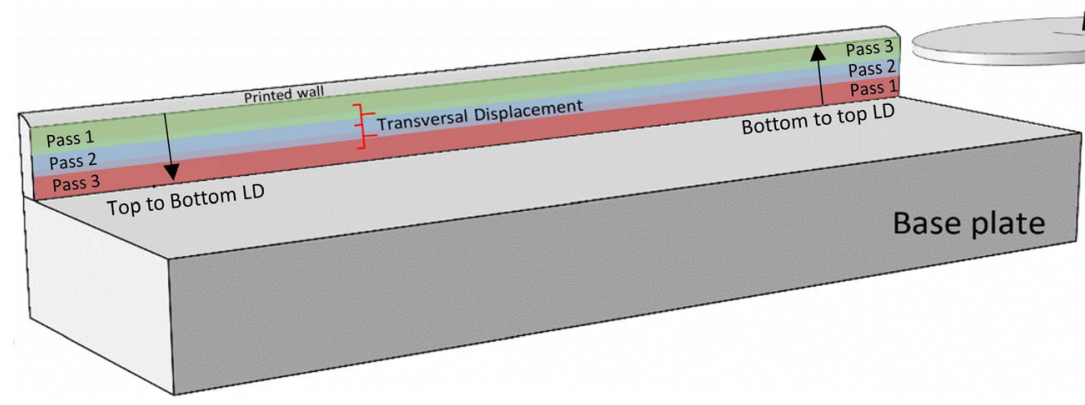

Fig. 9 Schematic demonstration of the transversal and lateral direction in the rolling process

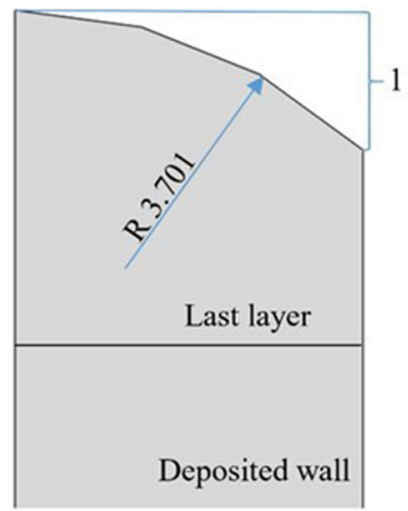

(a)
$1 \quad$ Location in which stresses are measured

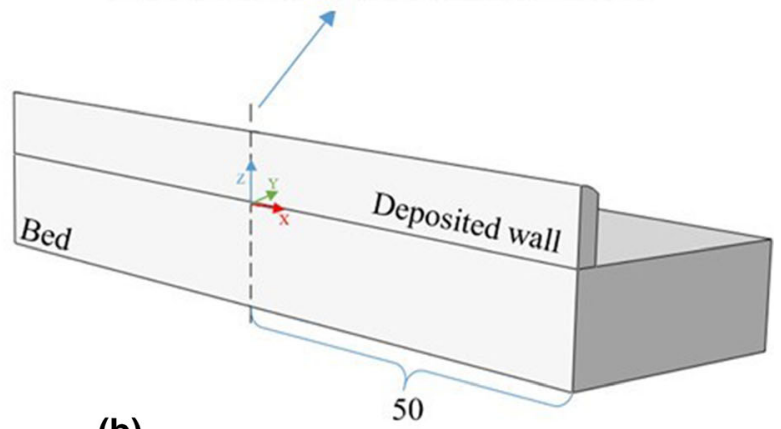

(b)

Fig. 10 The location of the residual stress profiles extracted from the simulated WAAM part (a) cross-sectional view, (b) 3D view

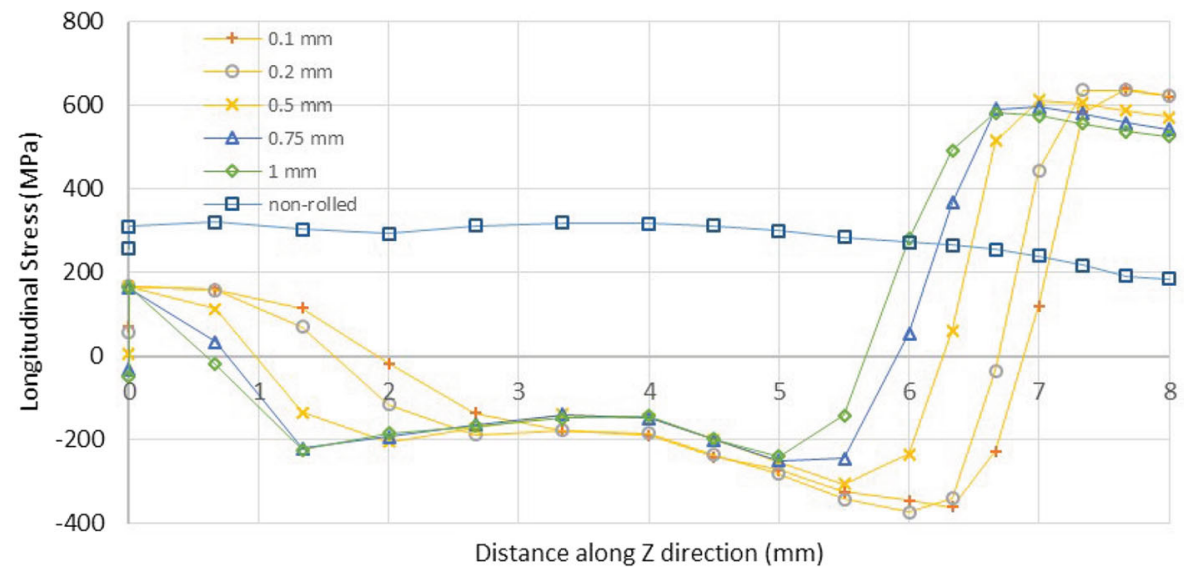

Fig. 11 Longitudinal stresses in the wall for different rolling depths

residual stress compared to the other two roller types, therefore making it the most effective roller type. Comparing the other two roller types, it can be observed that the magnitude of tensile residual stress using roller $\mathrm{A}$ is smaller and it appears in a narrower part of the bottom layers in the WAAM wall, compared to roller $\mathrm{C}$. This is believed to be due to the flat contact surface in roller $\mathrm{C}$, which provides a poor contact surface with the WAAM part. Finally, it is seen in Fig. 16 that none of the roller types appears to enable eliminating the stress plunge near the top layer of the wall, suggesting that this problem can only be solved by altering the RD.

\subsection{Pinch Rolling Process}

4.2.1 Transversal Displacement. The longitudinal and normal stress prediction results for pinch rolling simulations with TD values of 3, 3.5 and $4 \mathrm{~mm}$ are presented in Fig. 17 and 18, respectively. Figure 17 shows that a similar trend for longitudinal stress can be observed for different TD values. Moreover, this figure shows that compressive stresses are induced into almost the entire height of the wall with the peak values observed at the top layer. The effect of variation in TD 


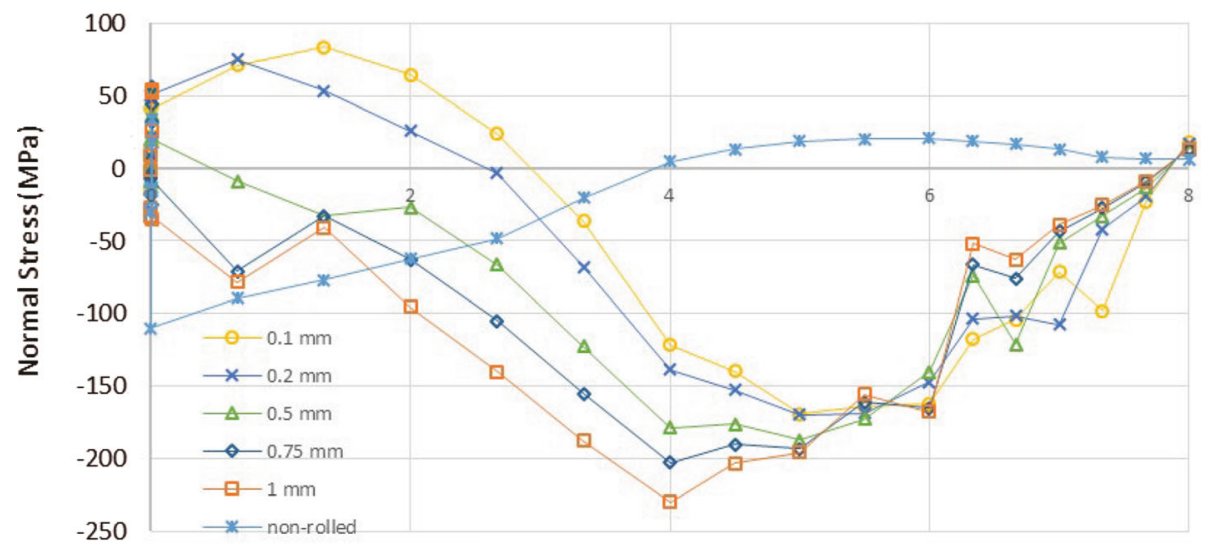

Distance along Z direction ( $\mathrm{mm}$ )

Fig. 12 Normal stresses in the wall for different rolling depths

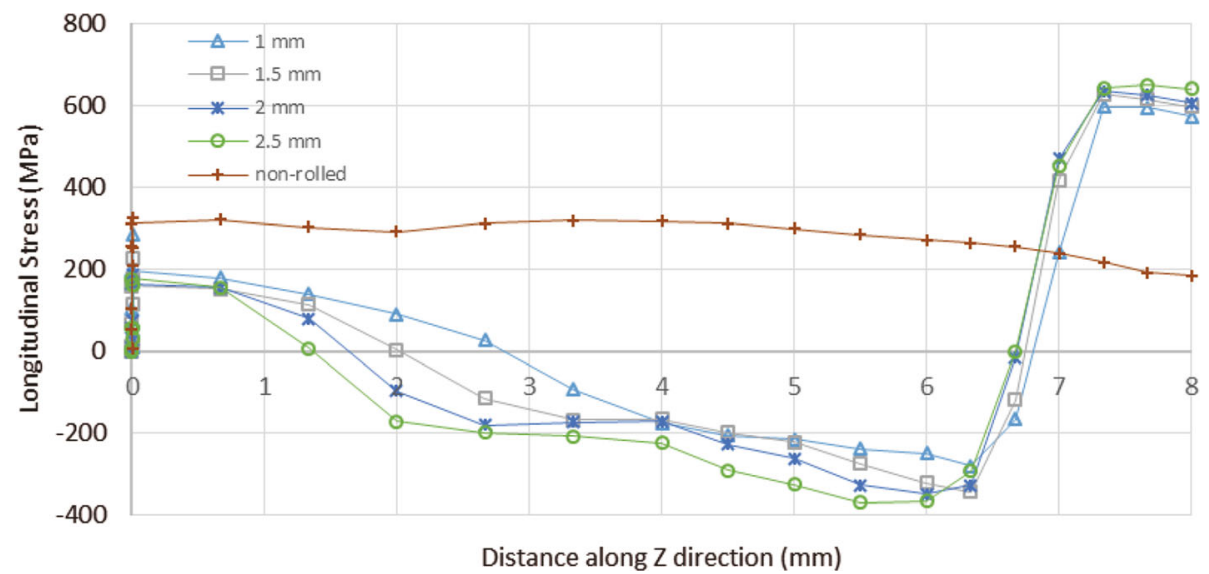

Fig. 13 Longitudinal stresses in the wall for different curvature depths

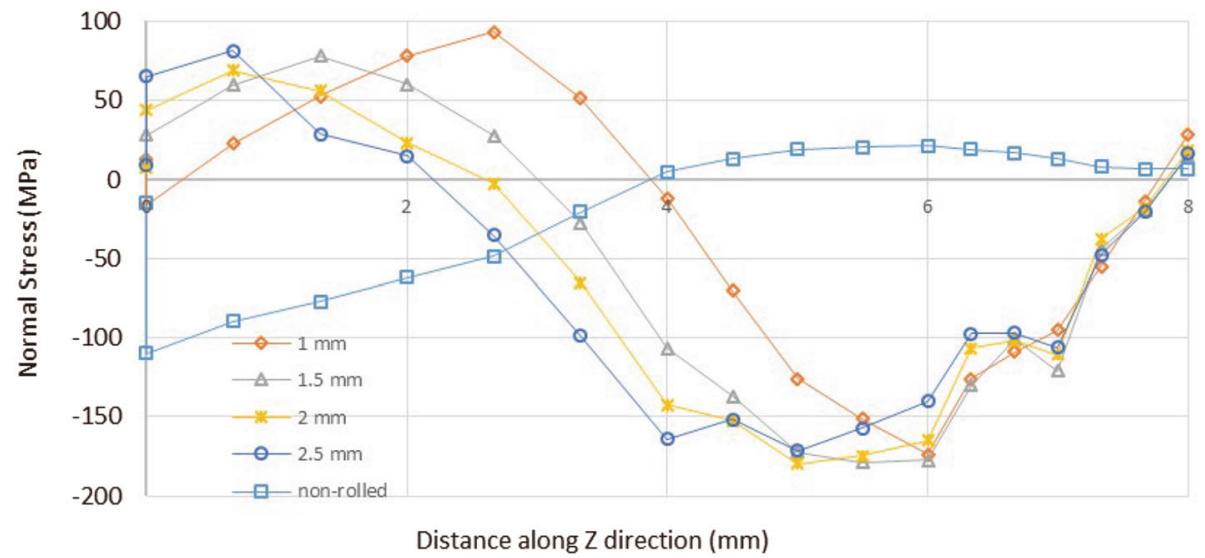

Fig. 14 Normal stresses in the wall for different curvature depths

value on the longitudinal residual stress appears to become negligible in the vicinity of the bedplate, which is due to the bedplate being constrained at the bottom of the wall.

Although the longitudinal stresses became compressed by pinch rolling, tensile stresses are formed in the WAAM wall along the normal direction (see Fig. 18). It can be seen in Fig. 18 that a smaller TD results in a larger proportion of the wall face being rolled twice, therefore slightly shifting down the tensile residual stress profile compared to the roller with the largest TD value. Pinch rolling results, however, indicate no abrupt change in the normal residual stresses through the wall depth. The normal residual stresses appear to be almost the same near the top of the wall, where no rolling was applied. 


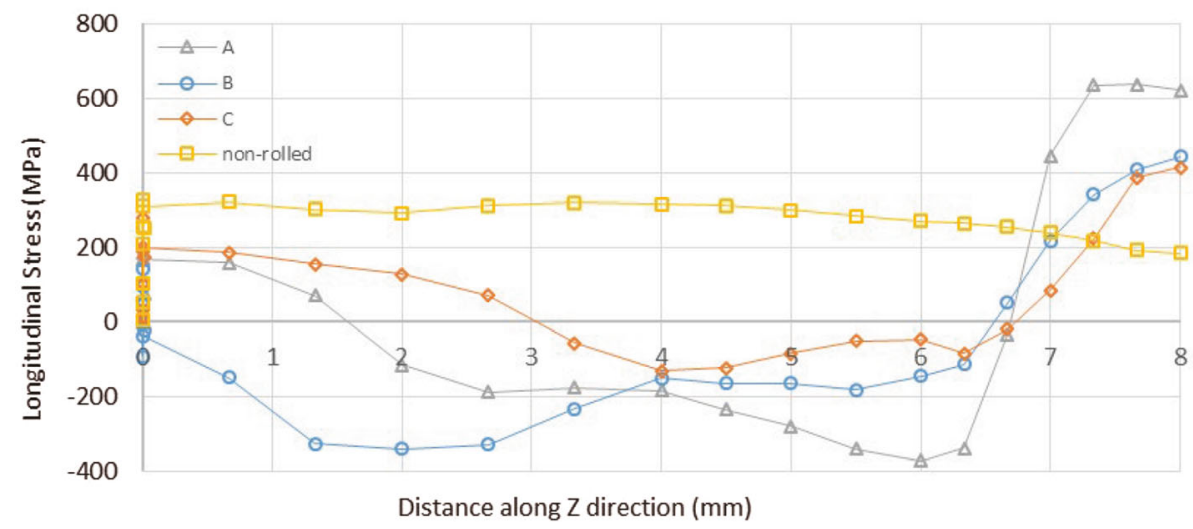

Fig. 15 Longitudinal stresses in the wall for different roller types

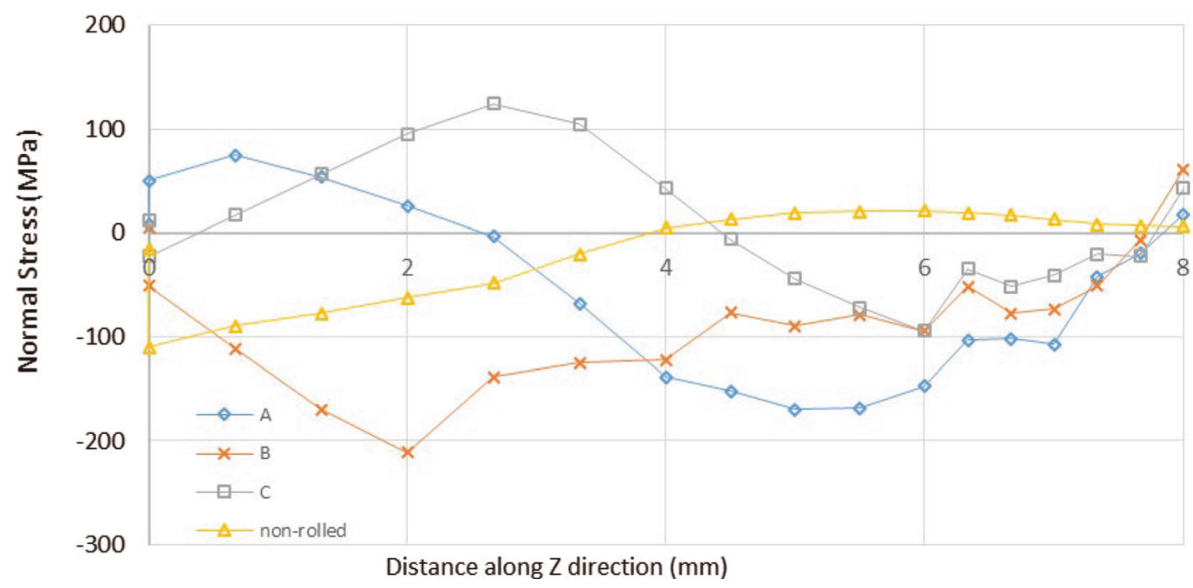

Fig. 16 Normal stresses in the wall for different roller types

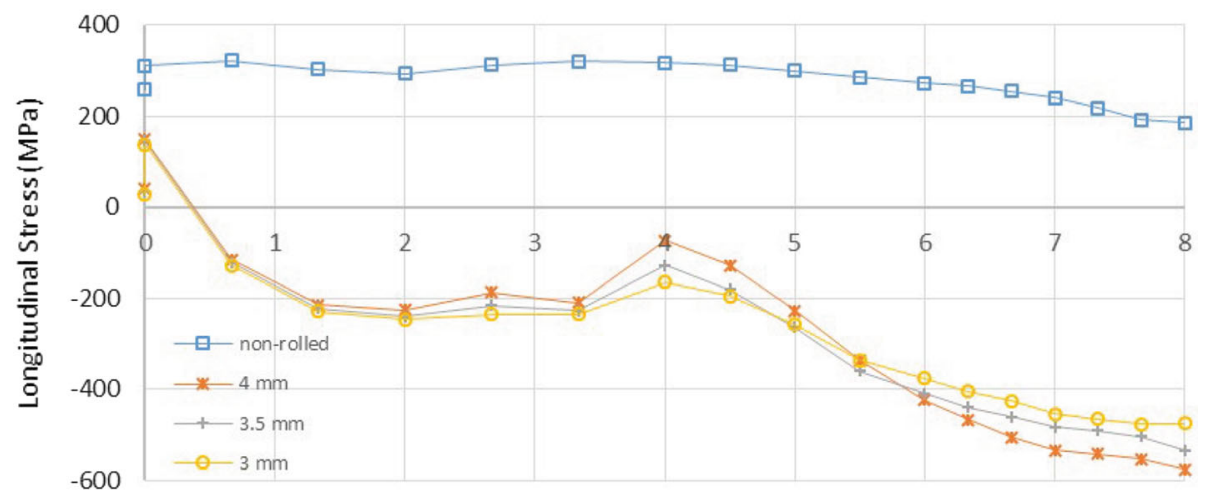

Distance along Z direction ( $\mathrm{mm}$ )

Fig. 17 Longitudinal stresses in the wall for different transversal displacements

4.2.2 Lateral Direction. The numerical results for investigating the LD on residual stress profiles are presented in Fig. 19 and 20 for longitudinal and normal stress directions, respectively. As seen in Fig. 19, the distribution of longitudinal residual stresses is shown to be affected by a change in the LD, which is determined by whether the rolling process was performed from the bottom to the top of the wall or in the reverse direction. In both cases, an abrupt increase in the magnitude of stress is observed in the vicinity of the bedplate.
The "top to bottom" process appears to be more effective, as it puts a relatively larger proportion of the material under compression, especially towards the bottom layers of the WAAM wall. Additionally, a more uniform distribution of longitudinal residual stresses is achieved in this rolling approach.

Figure 20 shows that a larger increase in the magnitude of the tensile normal residual stresses can be observed in the "top to bottom" case. The results show that the normal residual stresses start to decrease above a height of $3 \mathrm{~mm}$ using this 


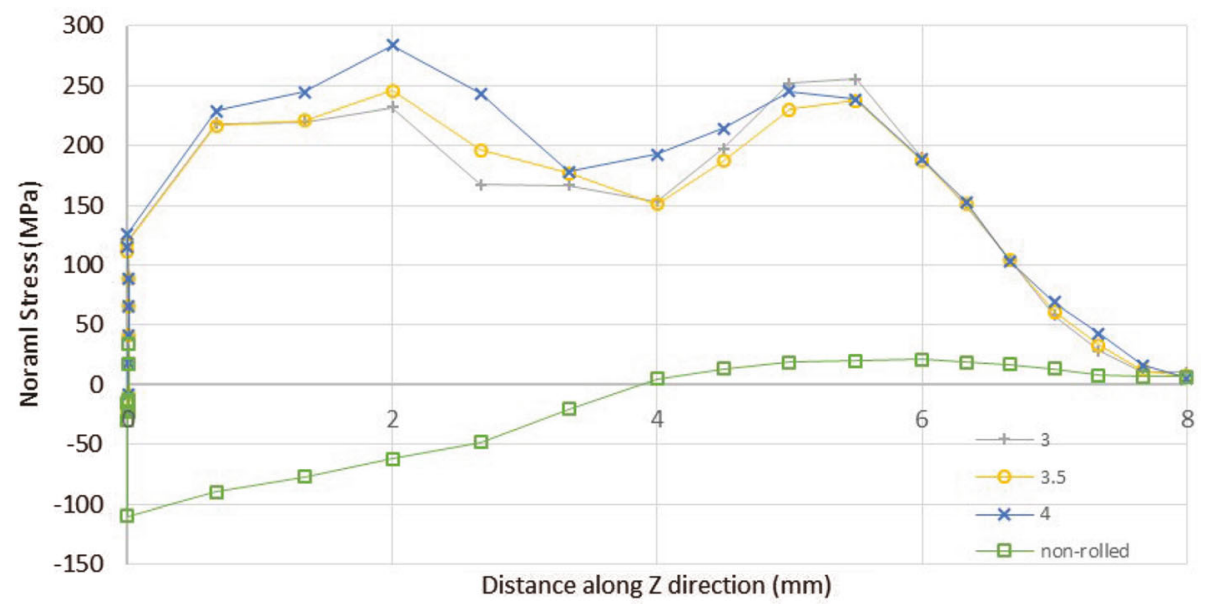

Fig. 18 Normal stresses in the wall for different transversal displacements

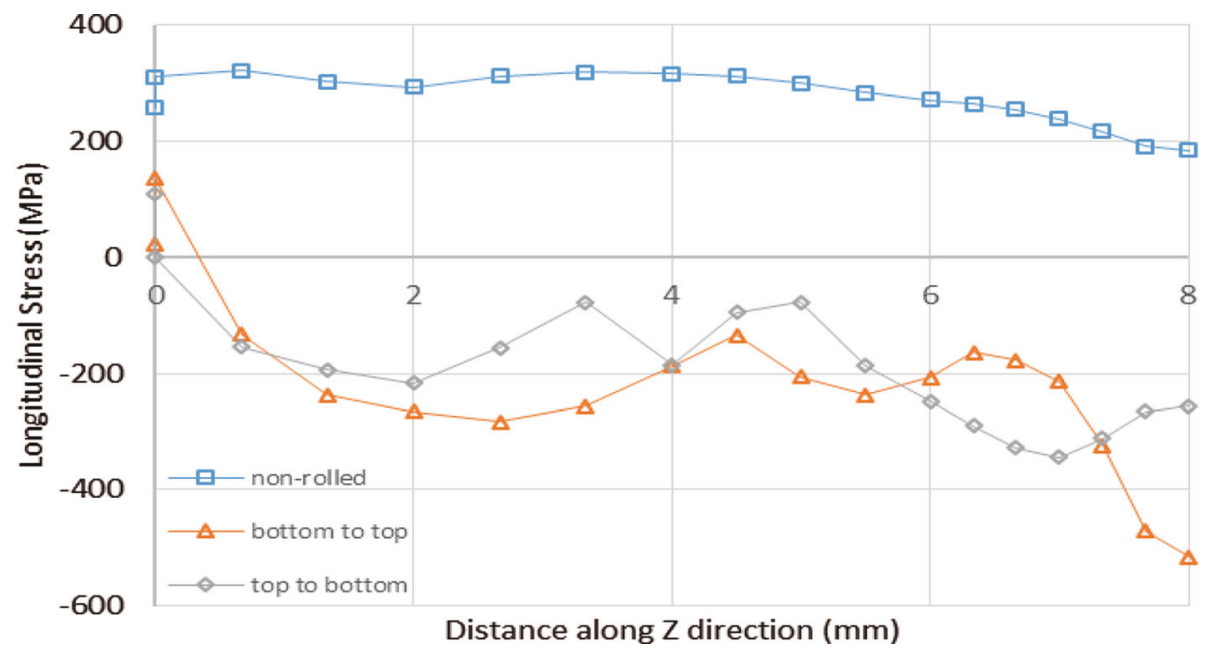

Fig. 19 The effect of lateral direction on longitudinal stresses in the wall

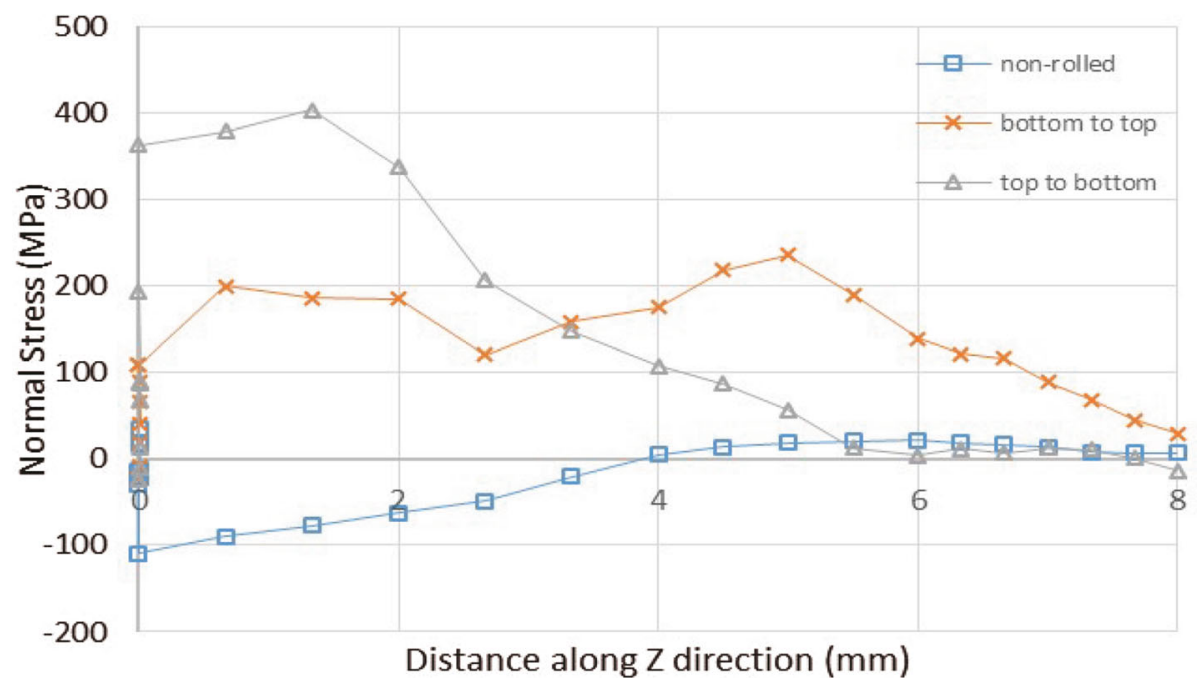

Fig. 20 The effect of lateral direction on normal stresses in the wall

process. The results also show that a more uniform distribution of the residual stresses is achieved using the "bottom-to-top" LD configuration. This was, however, not sufficient to elim- inate the tensile residual stresses created by the roller. The "top to bottom" process induces a large amount of normal residual 


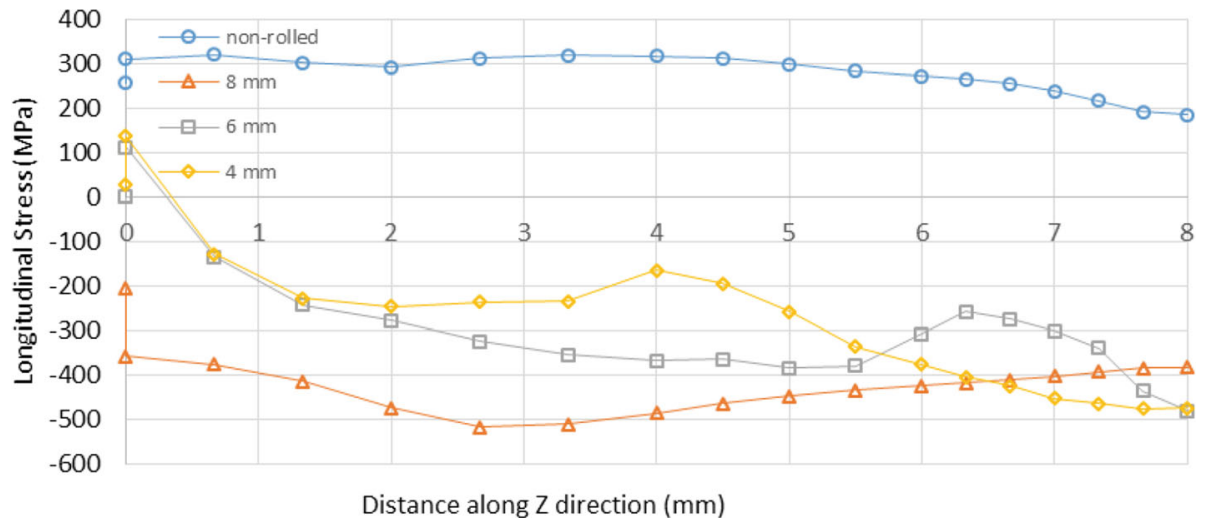

Fig. 21 Longitudinal stresses in the wall for different roller thicknesses

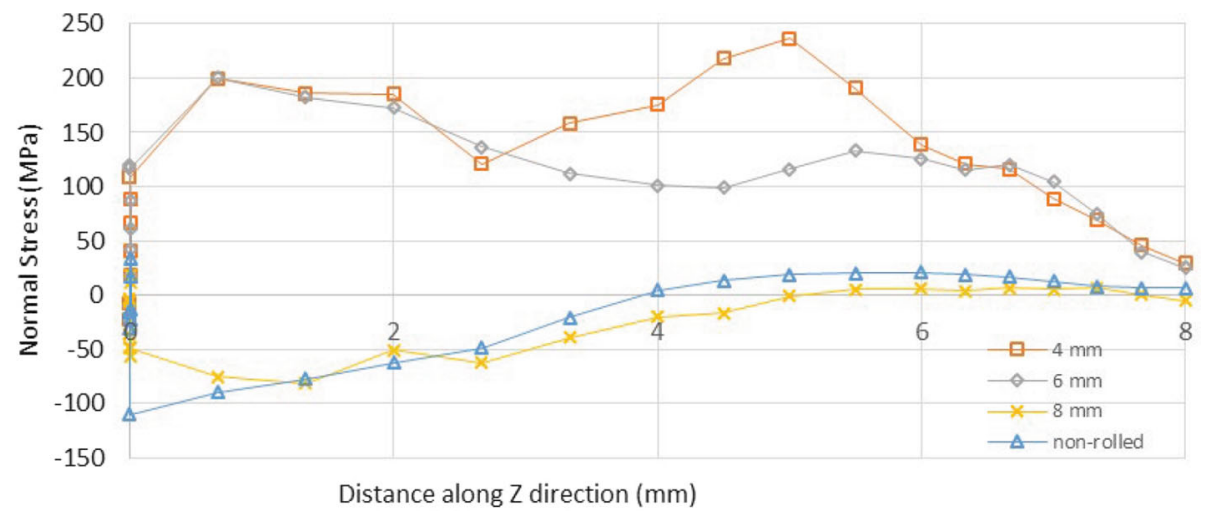

Fig. 22 Normal stresses in the wall for different roller thicknesses

stresses, which is caused by the bedplate constraining the wall from the bottom.

4.2.3 Roller Thickness. The numerical results for investigating the roller thickness effects on residual stress profiles are presented in Fig. 21 and 22 for longitudinal and normal stress directions, respectively. As seen in Fig. 21, more uniform stress distribution and a larger magnitude for the compressive longitudinal stresses throughout the wall are obtained using the $8 \mathrm{~mm}$ thick roller, which is the thickest roller considered in this study. One-pass rolling, using the thickest roller, appears to reduce the longitudinal tensile stresses by approximately $600 \mathrm{MPa}$. For the small and medium roller thicknesses, the maximum tensile stresses were created along the edges of the rollers. For the thickest roller, however, this was not observed, as the edge of the roller did not have contact with the wall face due to its thickness being larger than the wall height.

The results in Fig. 22 show that normal residual stresses do not seem to be affected by the small- and medium-sized roller thicknesses. Applying the one-pass rolling process using the $8 \mathrm{~mm}$ thick roller though introduces a predominantly compressive residual stress profile into the WAAM wall similar to that observed for longitudinal stresses. Moreover, the wall distortion appears to be eliminated through the one-pass rolling process, as it covers the entire wall face evenly.

\section{Conclusions}

The effects of vertical and pinch rolling processes on residual stress distribution in a WAAM wall have been investigated by means of finite element modelling. The model has been validated through comparison with the experimental residual stress measurements on the WAAM wall. According to the results presented in this study, the following conclusions can be drawn:

1. In the vertical rolling process, increasing the roller depth and curvature depth increases the beneficial compressive residual stresses penetration depth in the WAAM wall, particularly in the normal direction.

2. The curved-slotted roller induces a deeper and larger magnitude of compressive residual stresses in the WAAM wall compared to the profiled and flat-slotted rollers.

3. For the pinch rolling process, the results have shown that transversal displacements introduce dominantly compressive and entirely tensile stresses along the longitudinal and normal directions, respectively. 
4. The residual stress profile is sensitive to the pinch rolling direction, and it has been found that less damaging normal stresses are introduced into the WAAM wall using the "bottom-to-top" approach.

5. A larger magnitude of compressive residual stress is induced into the wall by applying a single pass using a thicker roller, compared to multiple passes using thinner rollers.

\section{Open Access}

This article is licensed under a Creative Commons Attribution 4.0 International License, which permits use, sharing, adaptation, distribution and reproduction in any medium or format, as long as you give appropriate credit to the original author(s) and the source, provide a link to the Creative Commons licence, and indicate if changes were made. The images or other third party material in this article are included in the article's Creative Commons licence, unless indicated otherwise in a credit line to the material. If material is not included in the article's Creative Commons licence and your intended use is not permitted by statutory regulation or exceeds the permitted use, you will need to obtain permission directly from the copyright holder. To view a copy of this licence, visit http://creativecommons.org/licenses/by/4.0/.

\section{References}

1. P.A. Colegrove, H.E. Coules, J. Fairman, F. Martina, T. Kashoob, H. Mamash et al., Microstructure and Residual Stress Improvement in Wire and Arc Additively Manufactured Parts Through High-Pressure Rolling, J. Mater. Process. Technol., 2013, 213, p 1782-1791

2. F. Montevecchi, G. Venturini, A. Scippa, and G. Campatelli, Finite Element Modelling of Wire-Arc-Additive-Manufacturing Process, Procedia CIRP, 2016, 55, p 109-114

3. S. Reddy, M. Kumar, J.S. Panchagnula, P.K. Parchuri, S.S. Kumar, K. Ito et al., A New Approach for Attaining Uniform Properties in Build Direction in Additive Manufactured Components Through Coupled Thermal-Hardness Model, J. Manuf. Process., 2019, 40, p 46-58

4. R. Sun, L. Li, Y. Zhu, W. Guo, P. Peng, B. Cong et al., Microstructure, Residual Stress and Tensile Properties Control of Wire-Arc Additive Manufactured 2319 Aluminum Alloy with Laser Shock Peening, J. Alloys Compd., 2018, 747, p 255-265

5. J. Ding, P. Colegrove, J. Mehnen, S. Ganguly, P.M. Sequeira Almeida, F. Wang et al., Thermo-Mechanical Analysis of Wire and Arc Additive Layer Manufacturing Process on Large Multi-Layer Parts, Comput. Mater. Sci., 2011, 50, p 3315-3322

6. J.D. Spencer, P.M. Dickens, and C.M. Wykes, Rapid Prototyping of Metal Parts by Three-Dimensional Welding, Proc. Inst. Mech. Eng. Part B J. Eng. Manuf., 1998, 212, p 175-182
7. D. Clark, M.R. Bache, and M.T. Whittaker, Shaped Metal Deposition of a Nickel Alloy for Aero Engine Applications, J. Mater. Process. Technol., 2008, 203, p 439-448

8. P.M. Almeida and S. Williams, Innovative Process Model of Ti-6Al4V Additive Manufacturing Using Cold Metal Transfer (CMT), in Proceedings of the 21st Annual International Solid Freeform Fabrication Symposium, 2010

9. B. Baufeld, O. Van der Biest, and R. Gault, Additive Manufacturing of Ti-6Al-4V Components by Shaped Metal Deposition: Microstructure and Mechanical Properties, Mater. Des., 2010, 31, p 106-111

10. B.A. Szost, S. Terzi, F. Martina, D. Boisselier, A. Prytuliak, T. Pirling et al., A Comparative Study of Additive Manufacturing Techniques: Residual Stress and Microstructural Analysis of CLAD and WAAM Printed Ti-6Al-4V Components, Mater. Des., 2016, 89, p 559-567

11. J. Zhang, X. Wang, S. Paddea, and X. Zhang, Fatigue Crack Propagation Behaviour in Wire + Arc Additive Manufactured Ti6Al-4V: Effects of Microstructure and Residual Stress, Mater. Des., 2016, 90, p 551-561

12. R. Li, J. Xiong, and Y. Lei, Investigation on Thermal Stress Evolution Induced by Wire and Arc Additive Manufacturing for Circular ThinWalled Parts, J. Manuf. Process., 2019, 40, p 59-67

13. J. Ding, P. Colegrove, J. Mehnen, S. Williams, P. Wang, and Almeida P. Sequeira, A Computationally Efficient Finite Element Model of Wire and Arc Additive Manufacture, Int. J. Adv. Manuf. Technol., 2014, 70, p 227-236

14. Y.A. Song, S. Park, and S.W. Chae, 3D Welding and Milling: Part II-Optimisation of the 3D Welding Process Using an Experimental Design Approach, Int. J. Mach. Tool. Manuf., 2005, 45, p 1063-1069

15. A.H. Nickel, D.M. Barnett, and F.B. Prinz, Thermal Stresses and Deposition Patterns in Layered Manufacturing, Mater. Sci. Eng., 2001, 317, p 59-64

16. M.P. Mughal, R.A. Mufti, and H. Fawad, The Mechanical Effects of Deposition Patterns in Weld-Based Layered Manufacturing, Proc. Inst. Mech. Eng. Part B J. Eng. Manuf., 2007, 221, p 1499-1509

17. T.L. Teng, P.H. Chang, and W.C. Tseng, Effect of Welding Sequences on Residual Stresses, Comput. Struct., 2003, 81, p 273-286

18. J.R. Hönnige, P.A. Colegrove, S. Ganguly, E. Eimer, S. Kabra, and S. Williams, Control of Residual Stress and Distortion in Aluminium Wire + Arc Additive Manufacture with Rolling, Addit. Manuf., 2018, 22, p 775-783

19. J. Ding, Thermo-Mechanical Analysis of Wire and Arc Additive Manufacturing Processes, Cranfield University Library, Bedford, 2012

20. J. Goldak, A. Chakravarti, and M. Bibby, A New Finite Element Model for Welding Heat Sources, Metall. Trans. B, 1984, 15(2), p 299-305

21. D. Forni, B. Chiaia, and E. Cadoni, Strain Rate Behaviour in Tension of S355 Steel: Base for Progressive Collapse Analysis, Eng. Struct., 2016, 119, p 164-173

22. M. Bijak-Żochowski and P. Marek, Residual Stress in Some ElastoPlastic Problems of Rolling Contact with Friction, Int. J. Mech. Sci., 1987, 39(1), p 15-32

23. F. Martina, M.J. Roy, B.A. Szost, S. Terzi, P.A. Colegrove, S.W. Williams et al., Residual Stress of As-Deposited and Rolled Wire + Arc Additive Manufacturing Ti-6Al-4V Components, Mater. Sci. Technol., 2016, 32(14), p 1439-1448

Publisher's Note Springer Nature remains neutral with regard to jurisdictional claims in published maps and institutional affiliations. 